\title{
openheart Assessment of exercise-induced changes in von Willebrand factor as a marker of severity of aortic stenosis
}

\author{
Jo M Zelis (D) , ${ }^{1}$ Frederik M Zimmermann, ${ }^{1}$ Froukje P J Andriessen, ${ }^{1}$ \\ Patrick Houthuizen, ${ }^{1}$ Jop Van de Ven, ${ }^{2}$ Jolanda Leuverman, ${ }^{2}$ Nils P Johnson, ${ }^{3}$ \\ Nico H J Pijls, ${ }^{1}$ Volkher Scharnhorst, ${ }^{2}$ Marcel Van 't Veer, ${ }^{1}$ Pim A L Tonino ${ }^{1}$
}

To cite: Zelis JM, Zimmermann FM, Andriessen FPJ, et al. Assessment of exercise-induced changes in von Willebrand factor as a marker of severity of aortic stenosis. Open Heart 2020;7:e001138. doi:10.1136/ openhrt-2019-001138

Received 19 July 2019 Revised 12 December 2019 Accepted 10 February 2020

Check for updates

(c) Author(s) (or their employer(s)) 2020. Re-use permitted under CC BY-NC. No commercial re-use. See rights and permissions. Published by BMJ.

${ }^{1}$ Cardiology, Catharina Hospital, Eindhoven, The Netherlands 'Laboratory, Catharina Hospital, Eindhoven, The Netherlands ${ }^{3}$ Division of Cardiology Department of Medicine, Weatherhead Pet Center McGovern Medical School at UTHealth and Memorial Hermann Hospital, Houston, Texas, USA

Correspondence to Dr Jo M Zelis; jo.zelis@ catharinaziekenhuis.nl

\section{ABSTRACT}

Background Loss of high-molecular-weight multimers (HMWMs) of von Willebrand factor (vWF) occurs due to high shear stress in patients with aortic stenosis. As symptoms of aortic stenosis occur during exercise, measurement of vWF during exercise might identify patients with aortic stenosis of clinical importance. The aim of this pilot study is to evaluate whether vWF changes over time as a result of exercise in patients with asymptomatic moderate or severe aortic stenosis.

Methods Ten subjects were analysed for changes in vWF by measuring HMWMs and closure time with adenosine diphosphate (CT-ADP). All subjects underwent a full stress test on a bicycle ergometer. At rest and at peak exercise, a transthoracic echocardiogram was performed. HMWMs and CT-ADP were assessed at baseline, during and after exercise.

Results HMWMs and CT-ADP did not change significantly during exercise, $p=0.45$ and $p=0.65$, respectively. HMWMs and CT-ADP correlated well, Spearman's rho $-0.621, p<0.001$. HMWMs during peak exercise did not correlate with maximal velocity measured, $p=0.21$. CTADP during exercise correlated well with the maximal echocardiographic velocity over the aortic valve (AV), rho $0.82, p=0.04$.

Conclusions In a cohort of 10 patients with moderate or severe aortic stenosis, we observed no significant change in vWF biomarkers during exercise. Peak CT-ADP during exercise showed a good correlation with peak AV velocity measured with echo. Although CT-ADP is an easy test to perform and could be an alternative for peak AV velocity measured during exercise, our results suggest that it can only detect large changes in shear stress.

\section{INTRODUCTION}

Aortic valve stenosis (AS) has become the most common valve disease in the developed world. ${ }^{12}$ When severe and symptomatic, the disease has a devastating course if left untreated. ${ }^{3-7}$ Treating severe, symptomatic patients with surgical aortic valve replacement and more recently transcatheter aortic valve implantation (TAVI) has had a major impact on survival. ${ }^{8-11}$ However, in patients with asymptomatic moderate or severe AS,

\section{Key questions}

What is already known about this subject?

- von Willebrand factor (vWF) has been used to identify paravalvular leakage after transcatheter aortic valve implantation (TAVI) procedures. As shear stress is high when severe aortic stenosis is present, it should return to normal after implantation of TAVI devices. However, this does not happen when paravalvular leakage is present. The high shear stress state persists, causing loss of high-molecularweight multimers and changes in closure time with adenosine diphosphate.

What does this study add?

- As symptoms of aortic valve stenosis occur during exercise and shear stress rises with exercise, we hypothesised that vWF could function as a predictor of symptoms and risk. First vWF changes during exercise had to be established. However, in this current study, no changes of vWF during exercise were observed, even in the slightest in this small population. This could be due to the high shear stress state these patients were already in.

How might this impact on clinical practice?

- In a cohort of 10 patients with asymptomatic moderate or severe aortic stenosis, there was no significant change in vWF detected during exercise. Our findings do not support the use of exercise-induced change in VWF as a biomarker of aortic stenosis severity in this patient group.

there is continued debate over which strategy is optimal: early intervention or watchful waiting. ${ }^{12} 13$

Currently, decisions about intervention for AS are based on assessments of severity performed under resting conditions. ${ }^{14}$ However, symptoms in patients with AS occur during exercise and measurements at rest inaccurately predict stress hemodynamics. ${ }^{15}$ In addition, measurements obtained during stress are potentially more valuable and accurate in predicting prognosis. ${ }^{1617}$ In a subset of patients with a low-flow state and AS, exercise 
can be simulated using dobutamine during echocardiographic assessment, but it is not standard of care in all patients. ${ }^{18} 19$

Biomarkers have played a role in numerous studies to help identify patients with higher risk $\mathrm{AS},{ }^{20}$ including use of NT-proBNP to predict outcome. ${ }^{21-23}$ Another biomarker is von Willebrand factor (vWF), which is known in AS for its role in Heyde's syndrome. ${ }^{24}{ }^{25}$ Due to high shear stress at the level of the aortic valve (AV), vWF undergoes proteolysis ${ }^{26-28}$ with resulting loss of high-molecular-weight multimers (HMWM) of vWF. This property has been used to identify aortic valve pathology. Another way to measure vWF function is a point-of-care measure of vWF-dependent platelet function, closure time with adenosine diphosphate (CT-ADP). In a recent study by van Belle $e t$ al, loss of vWF HMWM and a high value for CT-ADP were correlated with the presence of paravalvular regurgitation after TAVI and linked to higher mortality after 1 year. ${ }^{29}$ Both tests change in conditions with high shear stress and their results are highly dynamic, occurring within minutes after changes in blood flow, making it possible to use them as a biological mechanosensor. Other studies have linked vWF to the gradient over the aortic valve but none has found a definite link to prognosis and intervention. ${ }^{30}$

To our knowledge, no study has evaluated changes in HMWH and CT-ADP during exercise in patients with moderate AS with symptoms or asymptomatic severe AS. However, it is precisely these patients who pose the greatest challenge when it comes to identifying a subset that might benefit from early intervention. Given the highly dynamic character of the tests and the occurrence of symptoms during exertion, it would make physiological sense to identify patients at risk through the use of 'biomarker stress testing'. Therefore, the primary goal of this pilot study was to investigate if vWF biomarkers change during exercise and whether these changes correlate with transvalvular gradients in patients with asymptomatic moderate or severe AS.

\section{METHODS}

\section{Subjects}

A total of 10 subjects with asymptomatic moderate or severe AS were included between January and May 2017. Aortic stenosis severity was confirmed with echocardiography performed within 3 months before inclusion and classified as moderate (defined as valve area $1.0-1.5 \mathrm{~cm}^{2}$ or mean aortic gradient $25-40 \mathrm{~mm} \mathrm{Hg}$ in the presence of normal flow) or severe (defined as valve area $<1.0 \mathrm{~cm}^{2}$ or mean aortic gradient $>40 \mathrm{~mm} \mathrm{Hg}$ ). Exclusion criteria were non-revascularised and severe coronary artery disease, impaired left ventricular function (left ventricular ejection fraction $<50 \%$ ), significant concomitant valvular disease (stenosis or insufficiency of moderate or worse severity), coexisting hypertrophic cardiomyopathy, persistent atrial fibrillation or tachycardia with rapid ventricular response or active endocarditis. The local Institutional Review Board approved the study and all subjects gave written informed consent. This study was performed in compliance with the Declaration of Helsinki.

\section{Study protocol}

Age, sex, medical history, weight, height, medications, symptoms and quality of life using the advanced EQ-5D questionnaire were recorded at baseline. All subjects underwent a symptom-limited exercise test by pedalling a stationary bicycle ergometer. Expected heart rate and wattage were determined in advance based on age, sex and height. Every minute the workload increased by 20 W. Every 2 min, a 12-lead ECG and blood pressure (via arm cuff) were recorded. The exercise test was defined as abnormal in case of: intolerable symptoms during exercise (eg, angina, dizziness or syncope), drop in systolic blood pressure below baseline, signs of ischaemia on the ECG and/or ventricular tachycardia. Transthoracic echocardiography was performed three times. Once in the supine position, then while seated on the bicycle ergometer at rest and one final time at peak exercise. The examination included standard B-mode images in all available planes, Doppler pulsed wave, continuous wave (if possible) and colour Doppler analysis of aortic, mitral and tricuspid valves.

\section{HMW-multimer and CT-ADP analysis}

Blood samples were collected at baseline, 4 min after the start of exercise, during peak exercise and at 5, 10 and 30 min after exercise (figure 1) using peripheral venous access with a $3 \mathrm{~mm}$ needle in the arm or hand. From these blood samples, the HMW-multimer ratio of vWF (HMWMs) and CT-ADP were measured. HMWMs was defined as the relative amount of HMW-multimers (larger than 15-mers) in the sample compared with normal pooled plasma (NPP; standard human plasma) as previously described.$^{29}$ This ratio of HMW-multimers to normal pooled plasma equals 1 without any abnormalities and

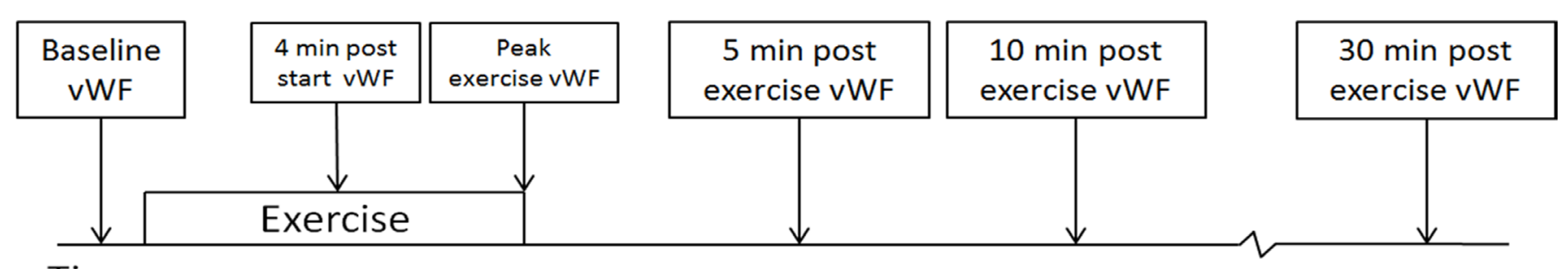

Time $\longrightarrow$

Figure 1 Timing of each blood sample during bicycle protocol. vWF, von Willebrand factor. 
becomes $<1$ when the HMW-multimer ratio is reduced. The CT-ADP was measured using the Platelet Function Analyzer (PFA)-100 (Siemens Medical Solutions Diagnostics, Netherlands), vWF multimers were analysed on a Hydrasys 2 system using 'Hydragel von Willebrand Multimers' and 'von Willebrand Multimers Visualisation' kits (all from SEBIA, Belgium). Analysis of CT-ADP with the point-of-care assay PFA-100 is used as a surrogate marker of vWF loss. Higher values correspond to a longer closure time and more loss of vWF. All assessments were done in our tertiary hospital laboratory with laboratory personnel blinded to clinical data.

\section{Statistical analysis}

Data are expressed as mean $( \pm \mathrm{SD})$ unless stated otherwise. For continuous variables with normal distribution and variance homogeneity, student's t-test and repeated measures analysis of variance were used as appropriate. For paired data without a normal distribution the Wilcoxon Signed Rank test and Friedman test (repeated measurements) were used. Dichotomous variables are expressed as absolute numbers and percentages (\%) and compared using the $\chi^{2}$ test or Fisher's exact test as appropriate. Spearman's Rank Order correlation was used to test the relation of continuous or nominal variables. A $p$ value less than 0.05 was considered statistically significant, and applicable tests were always two-sided. All analyses were conducted using SPSS 25 software (IBM, Armonk, New York, USA).

\section{RESULTS}

\section{Study population}

Table 1 provides the baseline characteristics of the 10 subjects. There were more men $(80 \%)$ than women, mean age was 62.7 years, three bicuspid aortic valves and two severely stenotic aortic valves at rest. All subjects had a good quality of life assessment score ranging from 7 to 9.5 for overall health.

\section{Echocardiography and ergometry}

Table 2 details the exercise and echocardiography data. All subjects ended the test due to fatigue, no adverse events occurred and none of the tests had signs of ischaemia. There was no other concomitant severe other valve disease and all subjects had normal left ventricular function. Eight of the 10 subjects finished the full ergometry cycling protocol(table 3). The systolic blood pressures rose for all subjects and exercise duration was 9 min on average. None of the subjects had cardiac symptoms, showed signs of ischaemia or experienced a blood pressure drop during cycling. The mean percentage increase in aortic valve velocity from rest to exercise was $14.5 \% \pm 5.8 \%$ (Wilcoxon signed rank, $\mathrm{p}=0.046$ ). In four subjects, no valid aortic valve velocity could be obtained during exercise.

\section{CT-ADP and HMWMs measurements}

The mean HMWMs and CT-ADP and the individual changes during all stages of the protocol are presented

\begin{tabular}{|c|c|}
\hline Variables & Summary $(n=10)$ \\
\hline Age (years) & $62.7 \pm 9.1$ \\
\hline Male $(n, \%)$ & $8(80 \%)$ \\
\hline BMI $\left(\mathrm{kg} / \mathrm{m}^{2}\right)$ & $27.7 \pm 2.9$ \\
\hline \multicolumn{2}{|l|}{ Risk factors } \\
\hline Active smoking $(n, \%)$ & $0(0 \%)$ \\
\hline Hypertension (n, \%) & $8(80 \%)$ \\
\hline Dyslipidaemia (n, \%) & $6(60 \%)$ \\
\hline Diabetes mellitus (n, \%) & $1(10 \%)$ \\
\hline \multicolumn{2}{|l|}{ Cardiac and vascular disease } \\
\hline Prior myocardial infarction (n, \%) & $1(10 \%)$ \\
\hline Bicuspid aortic valve $(n, \%)$ & $3(30 \%)$ \\
\hline History of peripheral vessel disease $(\mathrm{n}, \%)$ & $0(0 \%)$ \\
\hline History of COPD (n, \%) & $0(0 \%)$ \\
\hline \multicolumn{2}{|l|}{ Medication } \\
\hline Aspirin use (n, \%) & $1(10 \%)$ \\
\hline Statin use $(n, \%)$ & $5(50 \%)$ \\
\hline Diuretic use $(n, \%)$ & $3(30 \%)$ \\
\hline \multicolumn{2}{|l|}{ Symptoms } \\
\hline CCS score $>1$ (n, \%) & $0(0 \%)$ \\
\hline NYHA score $>1(n, \%)$ & $0(0 \%)$ \\
\hline Syncope $(n, \%)$ & $1(10 \%)$ \\
\hline \multicolumn{2}{|l|}{ Echocardiography } \\
\hline Moderate aortic stenosis (n, \%) & $7(70 \%)$ \\
\hline Ejection fraction at rest $(\%)$ & $66 \pm 4$ \\
\hline Mean pressure gradient $\mathrm{AV}(\mathrm{mm} \mathrm{Hg})$ & $32 \pm 3$ \\
\hline AVA $\left(\mathrm{cm}^{2}\right)$ & $1.0 \pm 0.9(n=9)$ \\
\hline AVA indexed $\left(\mathrm{cm}^{2} / \mathrm{m}^{2}\right)$ & $1.1 \pm 0.3(n=9)$ \\
\hline
\end{tabular}

Summary values represent number (\%) or mean \pm SD. $\mathrm{AV}$, aortic valve; AVA, aortic valve area measured with velocity time integral; BMI, body mass index; CCS, Canadian Cardiovascular Society grading of angina pectoris; COPD, chronic obstructive pulmonary disease; NYHA, New York Heart Association.

in figures 2 and 3, respectively. In two patients, analysis of the second stage failed due to insufficient blood samples. Both CT-ADP and HMWMs showed no significant change between rest and exercise, $\mathrm{p}=0.45$ and $\mathrm{p}=0.65$, respectively. Not all stages could be analysed for each patient. HMWMs and CT-ADP showed a negative correlation at rest (Spearman's rho $-0.74, \mathrm{p}<0.01$ ) and during exercise (Spearman's rho $-0.72, \mathrm{p}<0.02$ ). HMWMs did not correlate to the maximal transaortic velocity during exercise. CT-ADP during exercise correlated well with the maximal velocity measured with echo over the aortic valve (rho $0.82, \mathrm{p}=0.04$, figure 4 ). The correlation for CT-ADP at rest with the maximal velocity in rest did not correlate significantly (rho 0.61, $\mathrm{p}=0.059$ ). 


\begin{tabular}{ll}
\hline Table 2 Exercise data & \\
\hline Variables & Summary (n=10) \\
\hline $\begin{array}{l}\text { Exercise } \\
\text { Systolic blood pressure at rest (mm Hg) }\end{array}$ & $134 \pm 21$ \\
\hline $\begin{array}{l}\text { Systolic blood pressure during peak exercise } \\
\text { (mm Hg) }\end{array}$ & $185 \pm 21$ \\
\hline $\begin{array}{l}\text { Heart rate at rest (bpm) } \\
\text { Heart rate during peak exercise (bpm) }\end{array}$ & $73 \pm 12$ \\
$\begin{array}{l}\text { Percentage of predicted heart rate reached } \\
\text { (\%) }\end{array}$ & $92.4 \pm 10.6$ \\
$\begin{array}{l}\text { Percentage of predicated Wattage reached } \\
\text { (\%) }\end{array}$ & $102.6 \pm 19.7$ \\
\hline $\begin{array}{l}\text { \# of patients that did not finish the protocol } \\
\text { (n, \%) }\end{array}$ & $2(20 \%)$ \\
\hline $\begin{array}{l}\text { \# of patients with symptoms other than } \\
\text { fatigue during exercise (n, \%) }\end{array}$ & $0(0 \%)$ \\
\hline
\end{tabular}

Summary values represent number (\%) or mean \pm SD.

\section{DISCUSSION}

In this pilot study, we found that CT-ADP and HMWMs did not change significantly during exercise in patients with asymptomatic moderate or severe aortic stenosis. Nevertheless, peak CT-ADP during exercise showed a good correlation with peak AV velocity measured with echo while HMWM did not. Our data suggest that CT-ADP might be an easily available surrogate for peak transaortic pressure gradient during exercise and might therefore be a potential biomarker for prognosis.

Based on our results, measuring change in HMWMs with exercise does not seem to be a viable option to stratify the severity of aortic stenosis in asymptomatic patients. Measuring HMWMs is a particularly time-consuming process and because the correlation with CT-ADP (which is faster and easier to measure) is high, CT-ADP might be a valuable alternative for measuring vWF function. CT-ADP correlates well with echocardiographic peak velocity during exercise and might be more reliable to measure than maximal velocity with echo.

Notably, in 4 of the 10 subjects, no valid echo velocity could be obtained during peak exercise. Transthoracic

Table 3 Difference in rest and peak exercise of AV velocity, CT-ADP and loss of vWF HMWMs

\begin{tabular}{lcll}
\hline & $\begin{array}{l}\text { At rest } \\
(\mathbf{n}=10)\end{array}$ & $\begin{array}{l}\text { At peak } \\
\text { exercise }(\mathbf{n}=10)\end{array}$ & $\begin{array}{l}\mathbf{P} \\
\text { value }\end{array}$ \\
\hline $\begin{array}{l}\text { Maximal AV velocity } \\
(\mathrm{cm} / \mathrm{s})\end{array}$ & $359 \pm 56$ & $411 \pm 56(\mathrm{n}=6)$ & $0.046^{\star}$ \\
CT-ADP (sec) & $138 \pm 31$ & $138 \pm 65$ & $0.45^{\star}$ \\
HMWM ratio & $0.59 \pm 0.22$ & $0.61 \pm 0.17$ & $0.65^{\star}$ \\
\hline
\end{tabular}

*Wilcoxon Signed Rank test used; $\mathrm{p}<0.05$ was considered significant. Summary values represent number (\%) or mean \pm SD. $\mathrm{AV}$, aortic valve; CT-ADP, closure time with adenosine diphosphate; HMWMs, high-molecular-weight multimers; vWF, von Willebrand factor.

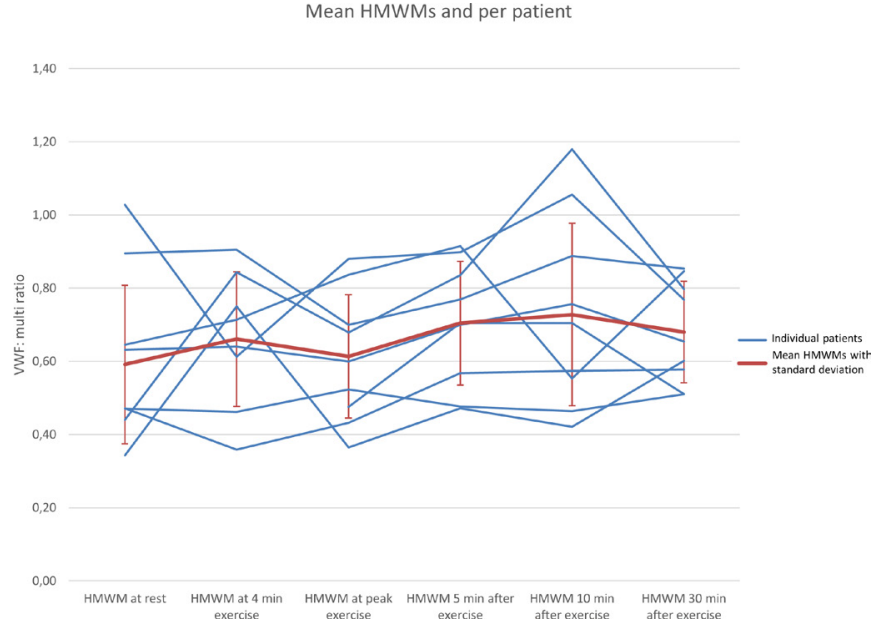

Figure 2 HMWM ratios to normal serum at each stage of the protocol showed a non-significant increase peaking 10 min after completion of exercise, with greater scatter among individual subjects vs the group mean $(n=8$, ANOVA pvalue 0.46). ANOVA, analysis of variance;HMWM; high molecular weight multimer;vWF, von Willebrand factor

echocardiography is not always an ideal tool to be used in an upright position while cycling. To find the highest gradient by echo, one can only use a limited number of echo views. Since most patients with AS experience symptoms during exercise and not during rest, it has been suggested in several studies that exercise testing is more important in the diagnostic work-up of such patients. ${ }^{173132}$ As echo gradient is difficult to acquire during exercise a CT-ADP might be a valuable alternative.

None of our patients showed any symptoms at rest and during exercise. This low-risk finding might indicate that stenosis anatomy was rather favourable and did not cause significant changes in shear stress, resulting in no important differences between rest and exercise for HMWM and CT-ADP in our cohort. In our study,

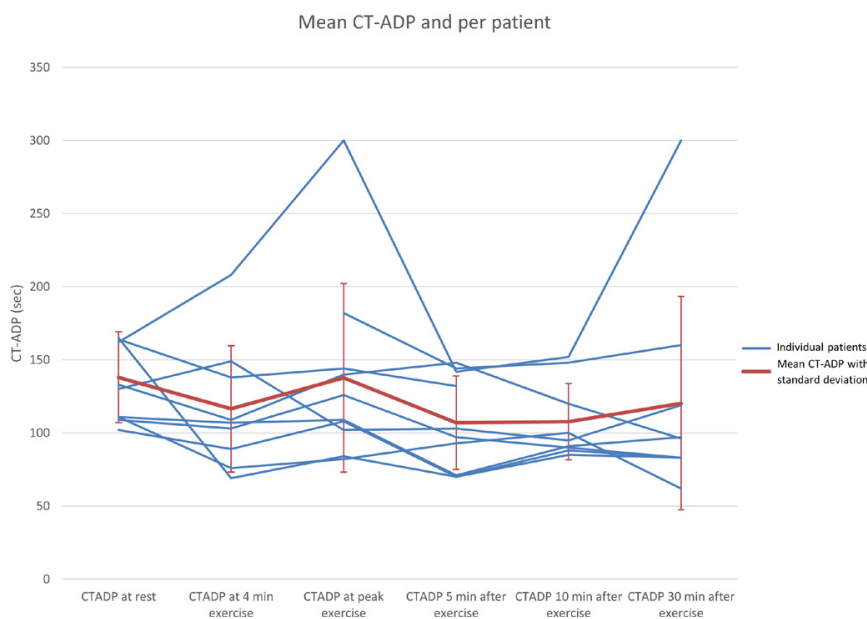

Figure 3 CT-ADP at each stage of the protocol showed a non-significant fall to a nadir 5-10 min after exercise, with greater scatter among individual subjects vs the group mean ( $n=8$, ANOVA pvalue 0.07).ANOVA, analysis of variance; CTADP, closure time with adenosine diphosphate. 


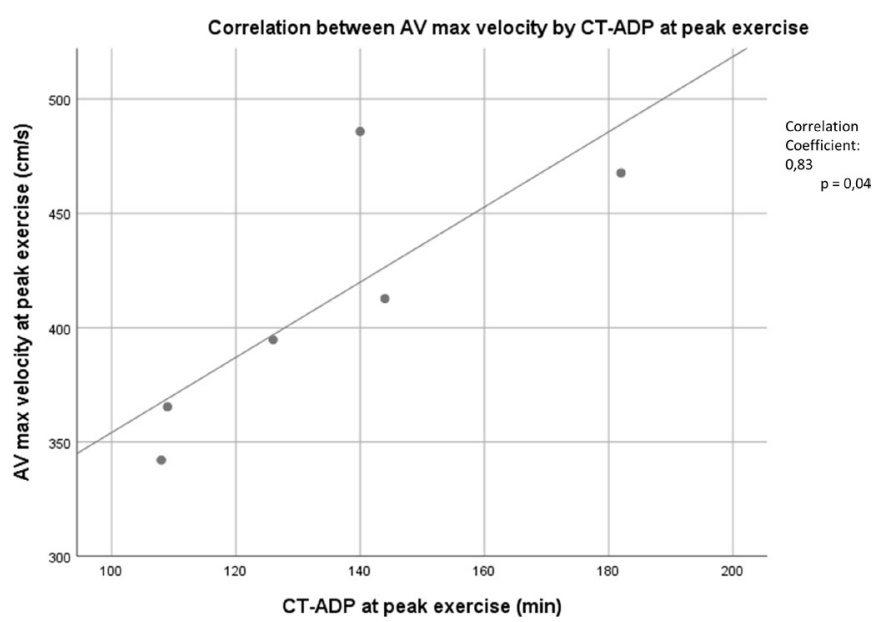

Figure 4 Maximal aortic valve velocity during peak exercise and CT-ADP showed a significant, direct correlation, rho 0.83, $p=0.04$ (Spearman's rho correlation). At peak exercise, only six subjects were eligible for echocardiography $(n=6)$. $\mathrm{AV}$, aortic valve; CT-ADP, closure time with adenosine diphosphate.

we went from an already high baseline shear stress state (moderate or severe aortic stenosis) to an even higher shear stress state with exercise. This increment might be too small to detect a biomarker difference. In contrast, a study by van Belle et al included subjects transitioning from a comparable high shear stress state (severe aortic stenosis) to a very low state of shear stress (after TAVI) and saw a significant difference in vWF breakdown products. Similarly, a different study performed with left ventricular assist devices saw significant changes in vWF levels during dramatic changes in shear stress. ${ }^{33}$

Prior work by Capoulade et al used a similar protocol to test the prognostic value of B-type natriuretic peptide levels after exercise. ${ }^{34}$ They found a prognostic value of peak-exercise BNP level as well as resting BNP levels. In their study, only patients with severe aortic stenosis and not moderate aortic stenosis were included. Our study did not look at long-term outcomes, but as there were no significant changes in vWF function during exercise, minimal prognostic effects would be expected.

Because we performed a pilot study, a small sample size was used. This may result in potential type 2 and 1 statistical errors. Furthermore, none of the patients experienced any symptoms at rest or during exercise, whereas symptomatic patients might have a more dramatic change in shear stress, and thus vWF, during exercise. Additionally, the study is too small to say anything about clinical follow-up.

The small lumen needle used to take blood samples might have caused some shear stress and therefore loss of vWF function. However, we estimate this potential effect to be small and moreover we did find differences in our TAVI cohort in which blood samples were drawn in the same way. Short-term experience in our hospital with HMWMs and CT-ADP in the post-TAVI setting showed fluctuations of the biomarker values in line with the results observed by van Belle et $a l,{ }^{29}$ suggesting a correct measurement protocol in our current study.

In patients with asymptomatic moderate or severe aortic stenosis, changes in HMWMs and CT-ADP during exercise were not significant. Peak CT-ADP during exercise showed a good correlation with peak AV velocity measured with echo. Although CT-ADP is an easy test to perform and could be an alternative for peak AV velocity measured during exercise, our results suggest that it can only detect large changes in shear stress, not modest changes as in this cohort.

Contributors All authors contributed significantly in this study and they have also read and approved the content of the manuscript. JMZ: design of the study, data acquisition and analyses and writing the paper. FMZ: design of the study and data acquisition and analyses. FPJA: data acquisition and analyses. PH: data acquisition and analyses. JVdV: data acquisition and analyses. JL: data acquisition and analyses. $\mathrm{NJ}$ : design of the study and verified the analytical methods. NHJP: funding and supervised the findings of this work. VS: supervised the data acquisition and analyses. MVV: design of the study data analyses and writing the article. PT: design of the study, funding and supervised the findings of this work.

Funding This investigator initiated study was supported by an unrestricted research grant from Medtronic Inc.

Competing interests None declared.

Patient consent for publication Not required.

Provenance and peer review Not commissioned; externally peer reviewed.

Data availability statement Data may be obtained from a third party and are not publicly available. All data relevant to the study are included in the article or uploaded as supplementary information.

Open access This is an open access article distributed in accordance with the Creative Commons Attribution Non Commercial (CC BY-NC 4.0) license, which permits others to distribute, remix, adapt, build upon this work non-commercially, and license their derivative works on different terms, provided the original work is properly cited, appropriate credit is given, any changes made indicated, and the use is non-commercial. See: http://creativecommons.org/licenses/by-nc/4.0/.

ORCID iD

Jo M Zelis http://orcid.org/0000-0003-2110-4901

\section{REFERENCES}

1 Lee H-F, Hsu L-A, Chan Y-H, et al. Prognostic value of global left ventricular strain for conservatively treated patients with symptomatic aortic stenosis. J Cardiol 2013;62:301-6.

2 Nkomo VT, Gardin JM, Skelton TN, et al. Burden of valvular heart diseases: a population-based study. Lancet 2006;368:1005-11.

3 Otto CM, Pearlman AS, Gardner CL. Hemodynamic progression of aortic stenosis in adults assessed by Doppler echocardiography. $J$ Am Coll Cardiol 1989;13:545-50.

4 Davies SW. Gershlick a H, Balcon R. progression of valvar aortic stenosis: a long-term retrospective study. Eur Heart $J$ 1991;12:10-14.

5 Gohlke-Bärwolf C, Minners J, Jander N, et al. Natural history of mild and of moderate aortic stenosis-new insights from a large prospective European study. Curr Probl Cardiol 2013;38:365-409.

6 Pellikka PA, Nishimura RA, Bailey KR, et al. The natural history of adults with asymptomatic, hemodynamically significant aortic stenosis. J Am Coll Cardiol 1990;15:1012-7.

7 Turina J, Hess O, Sepulcri F, et al. Spontaneous course of aortic valve disease. Eur Heart J 1987;8:471-83.

8 Dewey TM, Brown D, Ryan WH, et al. Reliability of risk algorithms in predicting early and late operative outcomes in high-risk patients undergoing aortic valve replacement. J Thorac Cardiovasc Surg 2008;135:180-7.

9 Salenger R, Gammie JS, Collins JA. Minimally invasive aortic valve replacement. J Card Surg 2016;31:38-50.

10 Smith CR, Leon MB, Mack MJ, et al. Transcatheter versus surgical aortic-valve replacement in high-risk patients. $N$ Engl J Med 2011;364:2187-98. 
11 Mack MJ, Leon MB, Smith CR, et al. 5-Year outcomes of transcatheter aortic valve replacement or surgical aortic valve replacement for high surgical risk patients with aortic stenosis (partner 1): a randomised controlled trial. Lancet 2015;385:2477-84.

12 Rosenhek R, Binder T, Porenta G, et al. Predictors of outcome in severe, asymptomatic aortic stenosis. $N$ Engl J Med 2000;343:611-7.

13 Généreux P, Stone GW, O'Gara PT, O'Gara PT, et al. Natural history, diagnostic approaches, and therapeutic strategies for patients with asymptomatic severe aortic stenosis. J Am Coll Cardiol 2016;67:2263-88

14 Baumgartner $\mathrm{H}$, Hung J, Bermejo J, et al. Recommendations on the echocardiographic assessment of aortic valve stenosis: a focused update from the European association of cardiovascular imaging and the American Society of echocardiography. Eur Heart $J$ Cardiovasc Imaging 2017;18:254-75.

15 Johnson NP, Zelis JM, Tonino PAL, et al. Pressure gradient vs. flow relationships to characterize the physiology of a severely stenotic aortic valve before and after transcatheter valve implantation. Eur Heart J 2018;39:2646-55.

16 Rafique AM, Biner S, Ray I, et al. Meta-Analysis of prognostic value of stress testing in patients with asymptomatic severe aortic stenosis. Am J Cardiol 2009;104:972-7.

17 Maréchaux S, Hachicha Z, Bellouin A, et al. Usefulness of exercisestress echocardiography for risk stratification of true asymptomatic patients with aortic valve stenosis. Eur Heart J 2010;31:1390-7.

18 Falk V, Baumgartner H, Bax JJ, et al. ESC/EACTS guidelines for the management of valvular heart disease. Eur $J$ Cardiothorac Surg 2017;2017:616-64.

19 Nishimura RA, Otto CM, Bonow RO, et al. 2017 AHA/ACC focused update of the 2014 AHA/ACC guideline for the management of patients with valvular heart disease: a report of the American College of Cardiology/American heart association Task force on clinical practice guidelines. Circulation 2017;135:2438-88.

20 Beckmann E, Grau JB, Sainger R, et al. Insights into the use of biomarkers in calcific aortic valve disease. J Heart Valve Dis 2010;19:441-52
21 Bergler-Klein J, Klaar U, Heger M, et al. Natriuretic peptides predic symptom-free survival and postoperative outcome in severe aortic stenosis. Circulation 2004;109:2302-8.

22 Clavel M-A, Malouf J, Michelena HI, et al. B-Type natriuretic peptide clinical activation in aortic stenosis: impact on long-term survival. $J$ Am Coll Cardiol 2014;63:2016-25.

23 Bergler-Klein J, Gyöngyösi M, Maurer G. The role of biomarkers in valvular heart disease: focus on natriuretic peptides. Can J Cardiol 2014;30:1027-34.

24 Vincentelli A, Susen S, Le Tourneau T, et al. Acquired von Willebrand syndrome in aortic stenosis. N Engl J Med 2003;349:343-9.

25 Smolen JS, Aletaha D, Koeller M, et al. New therapies for treatment of rheumatoid arthritis. Lancet 2007;370:1861-74.

26 Chapman WL, Rozenblit J, Bahill AT. System design is an NPcomplete problem. Syst Engin 2001;4:222-9.

27 Diacovo TG. Von Willebrand factor. Endothel Biomed 2007:915-21.

28 Hollestelle MJ, Loots CM, Squizzato A, et al. Decreased active von Willebrand factor level owing to shear stress in aortic stenosis patients. J Thromb Haemost 2011;9:953-8.

29 Van Belle E, Rauch A, Vincent F, et al. Von Willebrand factor multimers during transcatheter aortic-valve replacement. $N$ Engl J Med 2016;375:335-44.

30 Blackshear JL, Wysokinska EM, Safford RE, et al. Indexes of von Willebrand factor as biomarkers of aortic stenosis severity (from the Biomarkers of Aortic Stenosis Severity [BASS] study). Am J Cardiol 2013;111:374-81.

31 Magne J, Lancellotti P, Piérard LA. Exercise testing in asymptomatic severe aortic stenosis. JACC Cardiovasc Imaging 2014;7:188-99.

32 Henri C, Piérard LA, Lancellotti P, et al. Exercise testing and stress imaging in valvular heart disease. Can J Cardiol 2014;30:1012-26.

33 Nascimbene A, Neelamegham S, Frazier $\mathrm{OH}$, et al. Acquired von Willebrand syndrome associated with left ventricular assist device. Blood 2016;127:3133-41.

34 Biderman VL, Mart'yanova GV, Sorokin FD. Allowing for binder stiffness and reinforcement extensibility in designing a shell of revolution made of a composite material. Mech Compos Mater 1988;23:579-85. 